\title{
Fluctuations of the number of adsorbed molecules due to adsorption-desorption processes coupled with mass transfer and surface diffusion in bio/chemical MEMS sensors
}

\author{
Zoran Djurića, b ${ }^{\text {, Ivana Jokićc }}$, Adriana Peleša
}

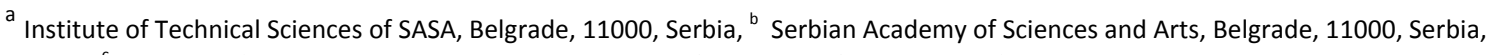
Institute of Chemistry, Technology and Metallurgy (IHTM-MTM), University of Belgrade, Belgrade, 11000, Serbia

Abstract: A comprehensive theoretical model of the fluctuations of the number of adsorbed molecules in MEMS bio/chemical sensors is presented for the first time; the model takes into account the processes of mass transfer, adsorption and desorption, and surface diffusion of adsorbed molecules. The numerical calculations performed using the derived theory show that the influence of surface diffusion on the fluctuations spectrum is significant and that it also depends on the species of adsorbed molecules.

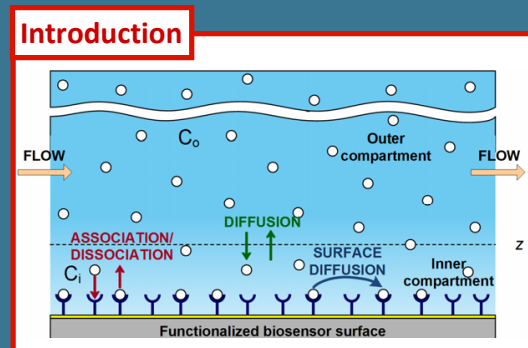

Processes in a sensor's reaction chamber and on the bio/chemical sensor surface.
In a large group of chemical and biological MEMS sensors, the output signal is related to the number of target molecules adsorbed on the active surface. Thus, the fluctuations in the number of adsorbed molecules inherently result in the sensor output signal fluctuations and limit sensor performance.

The processes in a sensor's reaction chamber which are relevant for the sensor's output signal generation or which affect the output signal:

- adsorption and desorption

- mass transfer (flow and diffusion)

- surface diffusion

They should be taken into account in the analysis of the fluctuations of the number of molecules adsorbed onto the sensor active surface.

\section{Theoretical derivations}

The variance of the number of adsorbed molecules and the "lifetime" of fluctuations, obtained by taking into account the mass transfer (the two-compartment model is used for the spatial distribution of the target molecules in the reaction chamber), respectively:

$$
\begin{aligned}
& <\Delta N^{2}>=\frac{k_{r} k_{f} C_{o}}{k_{r}+k_{f} C_{o}} N_{m} \tau \quad \tau=\frac{1}{k_{r}+k_{f} C_{o}}+\frac{k_{r} k_{f} N_{m}}{\left(k_{r}+k_{f} C_{o}\right)^{2} k_{m} A}-\frac{S(X)}{4<\Delta N^{2}>\tau}=\frac{1}{X^{2}}\left\{1-\frac{1}{2 \beta X}\left[(2-X) \sqrt{X+1}\left(1-e^{-\beta \sqrt{X+1}} \cos (\beta \sqrt{X-1})\right)\right.\right. \\
& \begin{array}{l}
\text { The diffusion equation (one } \frac{\partial \Delta N}{\partial t}=\frac{\partial}{\partial x}\left(D \frac{\partial \Delta N}{\partial x}\right)-\frac{\Delta N}{\tau} \\
\text { dimensional form): }
\end{array} \\
& X=\sqrt{1+\omega^{2} \tau^{2}} \quad \beta=\frac{L}{\sqrt{2 D \tau}}
\end{aligned}
$$

Using the "transport noise" approach for one-dimensional diffusion and generation-recombination processes, the spectral density of the fluctuations of the number of adsorbed particles is obtained:

\section{Numerical results}

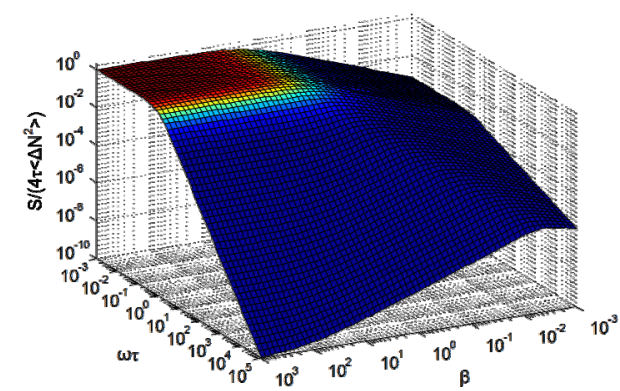

Normalized spectral density of the fluctuations in the number of adsorbed molecules as the function of the parameters $\beta=L /(2 D \tau)^{1 / 2}$ and $\omega \tau$. 3D diagram (left) and the cross-sections of the 3D diagram for five different values of $\beta$ (right).

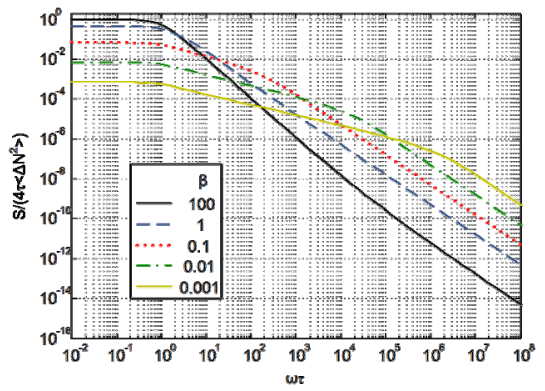

the

Conclusions

- The influence of surface diffusion on the spectrum of fluctuations of the number of adsorbed molecules is significant. For constant diffusion length $(D \tau)^{1 / 2}$, the influence becomes more prominent if the characteristic dimension (length) of the sensing surface is smaller.

- The shape of the measured noise spectrum reveals information about the analyte. Thus, it can be used for recognition of adsorbed species.

- The practical value of this study lies in the fact that the fluctuations of the number of adsorbed molecules can be a dominant noise component in affinity-based sensors. Therefore, the derived theory is useful in devising methods for the detection of analytes and the investigation of biomolecular interactions, based on frequency domain analysis of the measured fluctuations, as a complementary approach to time-domain measurements. It can also be used for estimation of sensor's limiting performance. 